\title{
Assessment of the clinical utility of serial $\beta$-D-glucan concentrations in patients with persistent neutropenic fever
}

\section{Correspondence \\ Michael Ellis \\ michael.ellis@uaeu.ac.ae}

Received 27 June 2007

Accepted 13 November 2007

\section{Michael Ellis, ${ }^{1}$ Basel al-Ramadi, ${ }^{2}$ Malcolm Finkelman, ${ }^{3}$ Ulla Hedstrom, ${ }^{4}$ Jorgen Kristensen, ${ }^{5}$ Hussein Ali-Zadeh ${ }^{5}$ and Lena Klingspor ${ }^{6}$}

\author{
${ }^{1}$ Department of Medicine, Faculty of Medicine and Health Sciences, UAE University, Al-Ain, UAE \\ ${ }^{2}$ Department of Medical Microbiology, Faculty of Medicine and Health Sciences, UAE University, \\ Al-Ain, UAE \\ ${ }^{3}$ Associates of Cape Cod, Falmouth, MA, USA \\ ${ }^{4}$ Department of Medicine, Al-Ain Hospital, Al-Ain, UAE \\ ${ }^{5}$ Department of Oncology, Tawam-Hopkins Hospital, AI-Ain, UAE \\ ${ }^{6}$ Department of Clinical Bacteriology, Karolinska University Hospital, Stockholm, Sweden
}

The performance of the Fungitell assay was investigated in 100 patients with haematological malignancy undergoing chemotherapy who developed antibiotic-unresponsive neutropenic fever (AUNF). Serum $\beta$-D-glucan (BG) concentrations were significantly elevated on the first day of AUNF and all subsequent alternate days to day 10 in 38 patients who developed an invasive fungal infection (IFI) compared to 42 patients remaining free of such infections. The mean and median values of BG were $171.9 \pm 29.6$ and $95.8 \mathrm{pg} \mathrm{ml}^{-1}$, respectively, for patients with IFI and $64.4 \pm 17.1$ and $32.9 \mathrm{pg} \mathrm{ml}^{-1}$ for patients with only AUNF $(P<0.0001)$. The differences remained significant over the 10 days despite antifungal therapy. The occurrence of $\geqslant 2$ sequential concentrations of $\geqslant 80 \mathrm{pg} \mathrm{ml}^{-1}$ ('positive' test) was found to give the best overall option for diagnosis, with an accuracy of $81.3 \%$, sensitivity of $86.8 \%$, positive predictive value of $76.7 \%$ and negative predictive value of $86.5 \%$. Of the patients with an IFI, $78 \%$ developed a positive test at or before the clinical diagnosis was made - this occurred at a mean (range) of 1.25 ( -14 to +14 ) days prior to the IFI diagnosis. By starting sampling of blood from the first day of neutropenia rather than from the first day of AUNF, $50 \%$ of the patients with subsequent IFI would have been identified 5 days earlier. Increasing sampling to daily from alternate-day frequency did not further improve this earlier timing of an IFI diagnosis. A greater proportion of patients with persistent high levels of $B G$ without overt IFI had severe enterocyte damage or mucositis than those with lower levels of BG without IFI $(P=0.002)$. If the results of the initial BG test had been acted on to change antifungal therapy, discontinuation would have been inappropriate in $30 \%$ of patients and would have delayed definitive antifungal therapy. Although the findings for the cohort of patients studied are very useful, there is inter-patient variability in the test's performance. An holistic diagnostic approach is therefore necessary to interpret the test results optimally. Future studies should address this in further detail as well as the impact of empirical antifungal drug use and patient outcome.

\section{INTRODUCTION}

Invasive fungal infections (IFI) and their attendant high mortality dictate that early, empirical antifungal therapy in

Abbreviations: AUNF, antibiotic-unresponsive neutropenic fever; BG, $\beta$ D-glucan; HRCT, high-resolution computed tomography; IFI, invasive fungal infection; IPA, invasive pulmonary aspergillosis; NPV, negative predictive value; PPV, positive predictive value.

Supplementary data are available with the online version of this paper. the persistently febrile neutropenic patient remains the practice standard (Pizzo et al., 1982; EORTC International Antimicrobial Therapy Cooperative Group, 1989; Walsh et al., 1999; Garey et al., 2006). Cost-effectiveness issues (EORTC International Antimicrobial Therapy Cooperative Group, 1989), adverse events and fungal resistance are valid concerns (Martino \& Viscoli, 2005) that have driven efforts to identify a subgroup of patients that would more reasonably benefit from antifungal treatment (De Pauw, 
2005). For example, HRCT detection of the halo sign has had a major impact on the outcome from invasive pulmonary aspergillosis and has high diagnostic specificity for Aspergillus (Caillot et al., 1997, 2001; Kami et al., 2002). Galactomannan, although originally heralded as a highly specific and sensitive diagnostic tool (Maertens et al., 2001), has not had the original high sensitivity claims subsequently confirmed (Pfeiffer et al., 2006). Nevertheless the potential usefulness of such serological tests has been shown when used as part of a wider diagnostic approach (Maertens et al., 2005). A large number of fungi other than Aspergillus species cause IFI in immunocompromised hosts (Fridkin, 2005), and therefore the Aspergillus-specific galactomannan test will fail to detect many fungal agents responsible for persistent neutropenic fever. The detection of $\beta$-D-glucan, which is a cell wall component of a wider range of fungi (Verweij et al., 2000; Odabasi et al., 2004; Ostrosky-Zeichner et al., 2005; Kelaher, 2006), would appear to be preferable in the setting of neutropenic fever.

However, the clinical experience with the Fungitell assay is somewhat limited (Odabasi et al., 2004; Ostrosky-Zeichner et al., 2005) and although the results support the usefulness of the assay, there is a need for further experience in clinical practice. Outstanding issues that need to be addressed include determining the optimal time of initiation of sampling, frequency of sampling and providing explanations for positive tests other than an early IFI. The clinical meaningfulness of a genuine positive test in the reality of the complex setting of a neutropenic patient with multiple reasons for fever is a further challenge. We therefore conducted this prospective study to evaluate the performance and clinical usefulness of the Fungitell assay in a cohort of patients who were not receiving antifungal prophylaxis. Patients in some other centres may receive antifungal prophylaxis and since its effect on glucan measurement is not known, our findings should be compared to such patient groups with caution.

\section{METHODS}

Study design. Patients eligible for inclusion into this study were expatriates living in the UAE, or UAE nationals, with haematological malignancy undergoing chemotherapy and developing neutropenia with fever unresponsive to broad-spectrum antibiotics for which they received antifungal therapy - see below.

It was anticipated that 100 consecutive patients who fulfilled the criteria for antibiotic-unresponsive neutropenic fever (AUNF - see below) and who gave their informed consent would be recruited. Recruitment commenced April 2004 and ended December 2005.

Serum samples were obtained from all patients on the first day (day 0 ) of AUNF and on subsequent alternate days for 14 days (7 estimations). Samples were stored at $-70{ }^{\circ} \mathrm{C}$ for up to 2 years prior to assay for $(1 \rightarrow 3)$ - $\beta$-D-glucan $(\mathrm{BG})$ by the Fungitell assay. The manufacturers of the assay kit state that samples can be stored for at least 6 years at such temperatures, even undergoing multiple freeze-thaw cycles without change in BG titres (personal communication, Associates of Cape Cod, Inc., Falmouth, MA, USA). Odabasi et al. (2004) used specimens stored for at least 6 years. Analysis was performed blinded to the clinical details. BG was measured when all samples had been collected.

In a subgroup of patients, sampling was performed daily from the first day of hospital admission irrespective of their initial neutropenic status, and throughout their hospital stay (see supplementary data, available with the online version of this paper).

BG assay. Serum BG was estimated using the Fungitell assay according to the manufacturer's instructions (Associates of Cape Cod). Frozen serum specimens were thawed, vortexed, and tested in triplicate. Five-microlitre aliquots were added to microplate wells and pre-treated for $10 \mathrm{~min}$ at $37^{\circ} \mathrm{C}$, with $20 \mu \mathrm{l}$ of an alkaline reagent $(0.125 \mathrm{M} \mathrm{KOH} / 0.6 \mathrm{M} \mathrm{KCl})$. One hundred microlitres of reconstituted Fungitell reagent was then added to the wells. The assay was monitored at $405 \mathrm{~nm}$, kinetically, for $40 \mathrm{~min}$ at $37^{\circ} \mathrm{C}$. Assay results (mean milli-absorbance units per minute) were interpolated against a standard curve run in each plate (reportable range $31-500 \mathrm{pg} \mathrm{ml}^{-1}$ ). Where results greater than $500 \mathrm{pg} \mathrm{ml}^{-1}$ are reported, the sample was diluted with glucan-free water to bring the results within the reportable range of the standard curve.

Patients. Patients were given appropriate chemotherapy according to internal guidelines (Coiffier et al., 2002; Medical Research Council Working Party on Leukemia in Adults, 2006; Farag et al., 2005).

No patient received antifungal drug prophylaxis. During neutropenia (neutrophils $<0.5 \times 10^{9} 1^{-1}$ ), at the onset of fever (single oral temperature of $\geqslant 38.3{ }^{\circ} \mathrm{C}$, or $\geqslant 38.0{ }^{\circ} \mathrm{C}$ for $\geqslant 1 \mathrm{~h}$ ) (Hughes et al., 2002) patients received standard empirical antibiotic therapy with piperacillin-tazobactam following sampling of three sets of blood cultures (Hughes et al., 2002). In patients with fever persisting for 3 days despite antibiotic treatment and sterile blood cultures, and no clinical focus for infection, and where there was no other explanation for the fever, the patient was deemed to be at high risk for IFI and received empirical treatment with either liposomal amphotericin B or caspofungin. All patients with AUNF included in this study therefore received empirical antifungal therapy. Concomitantly, a routine full diagnostic workup for IFI was commenced, including prolonged blood cultures for fungal pathogens, HRCT scan of chest and CT scan of liver, spleen and sinuses. This procedure was repeated weekly in all patients as long as AUNF persisted, or more frequently as indicated by new symptoms or signs. The diagnosis of IFI was made according to EORTC/MSG criteria (Ascioglu et al., 2002). However, galactomannan testing was not included in the diagnostic workup since a previous validation study in our unit indicated an unexpectedly low sensitivity for Apergillus infections. PCR diagnostics were not included for confirmation of the diagnosis of IFI for the purposes of the end points of this study. However, when PCR results were available they were referred to for interest only.

Inclusion criteria for patients were as follows: adult (at least 16 years), patients with haematological malignancy undergoing chemotherapy; becoming neutropenic $\left(<0.5 \times 10^{9} 1^{-1}\right)$ and developing fever; providing informed consent; on no antifungal prophylaxis; receiving broad-spectrum antibiotic treatment and either liposomal amphotericin B or caspofungin; having persistently negative bacterial blood cultures; having no radiological evidence of invasive fungal infection.

The Al Ain Medical District Human Research Ethics Committee gave approval for this study.

Statistical analysis. The mean and median BG concentrations in the subgroup of patients with IFI and AUNF alone were calculated. The two subgroups were compared on each alternate day with the Mann-Whitney test. $2 \times 2$ contingency tables were analysed with Fisher's exact test. Sensitivity, specificity, positive predictive value (PPV), negative predictive value (NPV) and accuracy were 
determined using standard definitions for cut-off values of BG of 60 , 80 and $100 \mathrm{pg} \mathrm{ml}^{-1}$.

\section{RESULTS}

\section{Demography and IFI}

From the 100 patients, 5 patient sample sets were discarded due to failure to collect a minimum of 4 sequential samples for analysis due to death (1 patient, due to haematological malignancy), withdrawal from the study (3 patients) or transfer to another medical unit ( 1 patient). The demographic data of the remaining 95 patients are shown in Table 1.

On the basis of clinical, radiographical and microbiological evidence as defined by EORTC/MSG criteria an IFI developed in 38 patients. AUNF only, without evidence of IFI, persisted in 42 patients. In 15 patients there was neither evidence of prolonged neutropenic fever nor IFI. Details of the IFI are shown in Table 2. There were only 5 cases of proven IFI - all candidaemia. No patient with invasive pulmonary aspergillosis (IPA) was a proven diagnosis because of preclusion of bronchoalveolar lavage and/or biopsy due to thrombocytopenia and/or poor performance status of the patient.

\section{Serial BG concentrations in AUNF and IFI}

A total of 572 serum specimens from alternate-day sampling were available for BG analysis: a mean of 6 samples per patient. Sequential patient sampling was complete for $\geqslant 96 \%$ anticipated samples from day 0 through day 8 . Sampling was complete for $78 \%$ from day 10 and day 12. Missing samples were due to resolution of AUNF, death, transfer to another unit or discharge home.

The serial BG concentrations for each patient who developed an IFI and for patients with AUNF without IFI are illustrated in Fig. 1. Mean and median values for each sampling point are shown in Table 3 . The values in patients with IFI were consistently significantly higher at the start of AUNF (day 0) and for each alternate day point through day
Table 2. Details of IFI

\begin{tabular}{|lcccc|}
\hline & Total & Proven & Probable & Possible \\
\hline Candidiasis & $\mathbf{1 4}$ & $\mathbf{5}$ & $\mathbf{7}$ & $\mathbf{2}$ \\
Candidaemia & 5 & 5 & 0 & 0 \\
Hepatosplenic & 6 & 0 & 6 & 0 \\
$\quad$ candidiasis & & & & \\
Disseminated & 3 & 0 & 1 & 2 \\
$\quad$ candidiasis & & & & \\
Aspergillosis & $\mathbf{2 4}$ & $\mathbf{0}$ & $\mathbf{1 4}$ & $\mathbf{1 0}$ \\
Pulmonary & 23 & 0 & 13 & 10 \\
Sinus & 1 & 0 & 1 & 0 \\
Total & $\mathbf{3 8}$ & $\mathbf{5}$ & $\mathbf{2 1}$ & $\mathbf{1 2}$ \\
\hline
\end{tabular}

${ }^{*}$ C. tropicalis 3 patients, C. albicans 1 , unspeciated Candida 1 . Three patients had candidaemia alone, one also had hepatosplenic candidiasis and one had pulmonary aspergillosis.

10. Over the 10 days, the median values in the AUNF group without IFI varied between 28 and $54 \mathrm{pg} \mathrm{ml}^{-1}$, compared to between 72.6 and $125.2 \mathrm{pg} \mathrm{ml}^{-1}$ in those patients developing an IFI. In the 15 patients with neither AUNF nor IFI the values were generally low and not significantly different from those for the AUNF alone group.

\section{Assay performance}

The performance of the test for cut-off values of between 60 and $100 \mathrm{pg} \mathrm{ml}^{-1}$ for 1 or $\geqslant 2$ consecutive values at these concentrations is shown in Table 4 . Defining a positive test in the presence of an IFI by $\geqslant 2$ consecutive concentrations of BG of $\geqslant 80 \mathrm{pg} \mathrm{ml}^{-1}$ gave a sensitivity, specificity, PPV, NPV and overall accuracy of $86.8 \%, 76.2 \%, 76.7 \%, 86.5 \%$ and $81.3 \%$, respectively, for alternate day sampling.

\section{Timing of the first positive test from alternate-day sampling from the first AUNF day}

Thirty-two of the 38 patients ( $84 \%$ ) with an IFI had a positive test by the fourth day of AUNF, of whom 28/38 (74\%) were positive on days 0 and 2 of AUNF. Twenty-eight

Table 1. Demographic data

\begin{tabular}{|lcccc|}
\hline & Total & IFI & AUNF alone & No IFI + no AUNF \\
\hline All patients & 95 & 38 & 42 & 15 \\
Male & 69 & 31 & 26 & 13 \\
Female & 26 & 7 & 16 & 2 \\
Malignancy & & & & 3 \\
AML & 49 & 19 & 28 & 12 \\
LL & 33 & 15 & 12 & 0 \\
Other & 13 & 4 & 2 & $32.3 \pm 11.9(16-58)$ \\
Age (years) & $34.8 \pm 11.4(16-58)$ & $32.8 \pm 10.9(16-58)$ & $35.7 \pm 11.7(16-58)$ & \\
\hline
\end{tabular}

*AML, acute myeloid leukaemia; LL, lymphocytic leukaemia; 'Other' includes chronic myelocytic leukaemia, Burkitt's lymphoma and Hodgkin's lymphoma. 

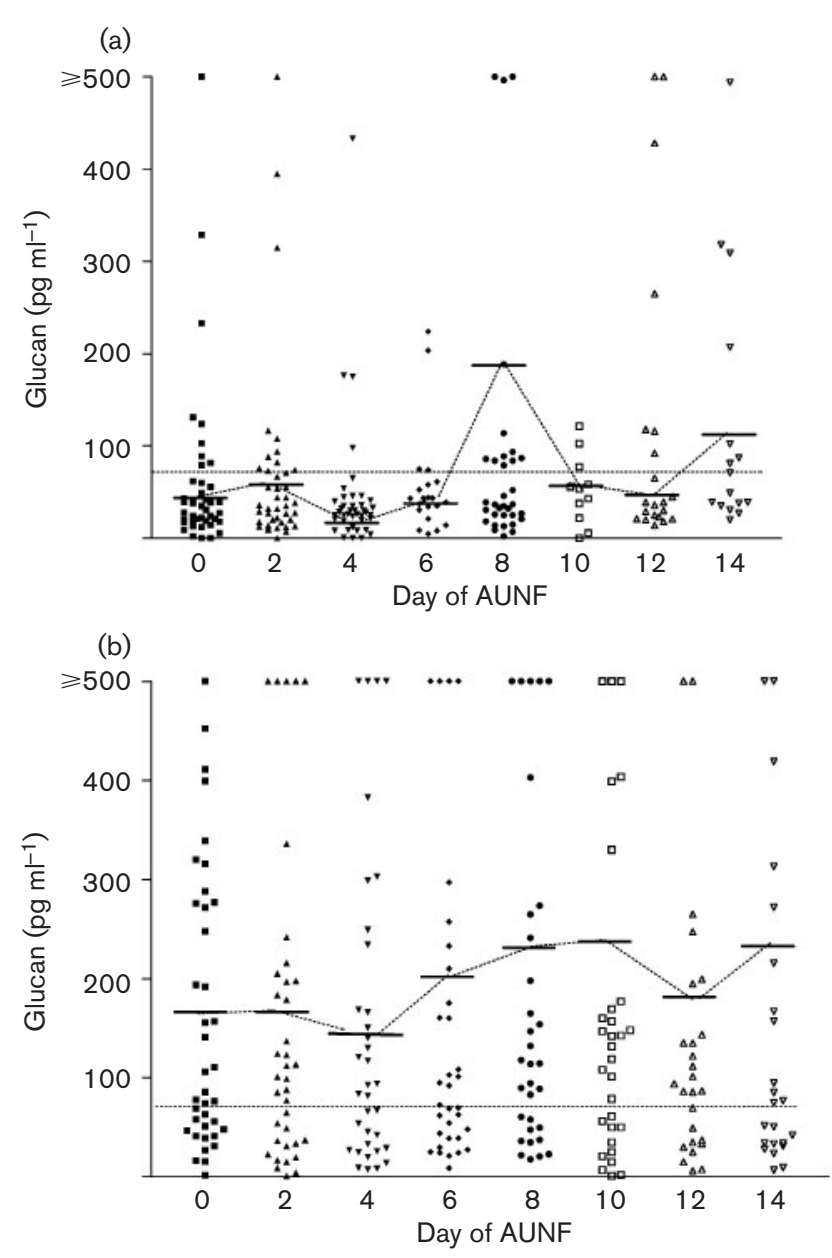

Fig. 1. Individual BG concentrations for patients with IFI (a) and AUNF (b). Mean values are depicted by the horizontal bars. The nominated cut-off value of $80 \mathrm{pg} \mathrm{ml}^{-1}$ is shown by the dotted line. See Table 3 for the full values.

of 36 patients $(78 \%)$ had a positive test at or before the first clinical and/or radiological signs of the IFI. The first positive test occurred a mean (range) of $1.25 \pm 1.0(-14$ to +14$)$ days prior to the IFI. The time to the first positive test relative to the day of AUNF is shown in Fig. 2(a) and the time of the first clinical diagnosis of IFI relative to the first positive BG test is shown in Fig. 2(b). Analysis of the preAUNF cohort indicated that positive BG results occurred 5 days prior to fever onset (see supplementary data, available with the online version of this paper).

\section{Patients with BG concentrations $>80 \mathrm{pg} \mathrm{ml}^{-1}$ without IFI}

In the 42 patients with AUNF who did not develop an IFI, 11 had $\geqslant 2$ consecutive BG readings of $\geqslant 80$ (median 54 ) pg ml $\mathrm{ml}^{-1}$ and 31 patients had only 1 reading of $\geqslant 80$ or readings $<80$ (median 31$) \mathrm{pg} \mathrm{ml}^{-1}(P<0.0001)$ (Table 5). In 8 of the 11 patients $(73 \%)$ with $\geqslant 2$ consecutive readings of $\geqslant 80 \mathrm{pg} \mathrm{ml}^{-1}$ there was either clinical (WHO oral mucositis toxicity grade $\geqslant 3)$ (2 patients) or radiological (CT/ultrasound gastrointestinal) evidence of intestinal enterocyte damage (enterocolitis) (3 patients) or the patients had a significant positive blood culture (3 patients) (Table 5). Three other patients had no abnormalities associated with the raised BG levels. Of the 31 patients with sporadic raised BG levels or whose BG levels were below $80 \mathrm{pg} \mathrm{ml}^{-1}$, only 6 (19\%) had either severe mucositis (1 patient) or a positive blood culture (5 patients) and 25 had no detectable abnormalities. The difference in the proportion of the patients with these abnormalities in the groups based on the BG readings was significant $(P=0.002)$. A typical time-course in such a patient with colitis and bacteraemia is shown in Fig. 3. Note the modestly high BG levels.

\section{Examples of variability in test profiles}

Four different patient examples are shown in Fig. 4(a-d). Fig. 4(a) represents a patient with IPA who developed neutropenic fever on day 7 . His BG assay was positive on day 4 , and rapidly reached a maximum of $490 \mathrm{pg} \mathrm{ml}^{-1}$ by day 8, despite antifungal therapy. Subsequently BG concentrations fell to $<80 \mathrm{pg} \mathrm{ml}^{-1}$ by day 16 of treatment. Fig. 4(b) is from a patient with enterocolitis during days 110 showing a high but brief rise in BG. IPA developed on day 22, when BG levels were moderately elevated. The levels fell with treatment and eventually reached $<80 \mathrm{pg}$ $\mathrm{ml}^{-1}$ when the IPA radiologically resolved (day 38). However, the patient had a further modest elevation of BG associated with abdominal pain. He also had a positive blood test for Candida mannan (Bio-Rad Platelia). Despite the fall in BG to very low levels $\left(<20 \mathrm{pg} \mathrm{ml}^{-1}\right)$ the patient died from underlying malignant disease and undetermined sepsis. Fig. 4(c) illustrates a patient with radiological deterioration of IPA despite falling BG levels. Note CT scan 1 , showing a halo sign on day 12 , and worsening of the lesions on day 20 (CT2). Fig. 4(d) is illustrative of high fluctuating BG levels, with probable IPA (halo sign and positive Aspergillus PCR).

\section{DISCUSSION}

We undertook this study to measure the performance of a BG assay among haematological patients in the Middle East undergoing chemotherapy, to describe sequential time changes in BG concentrations in patients who were originally naïve to antifungal prophylaxis, to anticipate the usefulness of knowing the real-time BG values at the start of AUNF, to investigate further the causes of high concentrations in patients without IFI, and to describe sequential changes in patients.

In the group of patients we investigated with AUNF who developed an IFI, BG concentrations, measured on the first day of AUNF and on subsequent alternate days, were found to be persistently and significantly higher compared to 
Table 3. BG concentrations for the first day of AUNF (day 0 ) and for each subsequent alternate day to day 14

Values shown are BG concentrations in $\mathrm{pg} \mathrm{ml}^{-1}$ as means \pm SEM, medians and $95 \%$ confidence intervals (CI). Differences for each alternate-day concentration between AUNF-only patients and patients with neither AUNF nor IFI are all non-significant. Refer to Fig. 1.

\begin{tabular}{|c|c|c|c|c|c|}
\hline $\begin{array}{l}\text { Day of } \\
\text { AUNF }\end{array}$ & Statistic & Patients with IFI & Patients with only AUNF & $\begin{array}{c}\text { Patients with neither IFI } \\
\text { nor AUNF }\end{array}$ & $\begin{array}{c}\text { Difference between IFI } \\
\text { and only AUNF }\end{array}$ \\
\hline \multirow[t]{2}{*}{0} & Mean & $171.9 \pm 29.6$ & $64.4 \pm 17.1$ & $41.5 \pm 11.1$ & \\
\hline & $95 \% \mathrm{CI}$ & $111.7-232.0$ & $29.7-99.1$ & $17.8-65.1$ & $P<0.0001$ \\
\hline \multirow[t]{2}{*}{2} & Mean & $179.2 \pm 36.2$ & $77.9 \pm 23.9$ & $60.6 \pm 14.1$ & \\
\hline & Median & 100.9 & 35.0 & 38.0 & \\
\hline \multirow[t]{3}{*}{4} & Mean & $208.7 \pm 54.0$ & $44.9 \pm 11.2$ & $87.8 \pm 24.6$ & \\
\hline & Median & 92.9 & 28.0 & 40.6 & \\
\hline & $95 \% \mathrm{CI}$ & $98.9-318.5$ & $22.1-67.7$ & $35.2-140.3$ & $P<0.0001$ \\
\hline \multirow[t]{3}{*}{6} & Mean & $209.7 \pm 64.0$ & $54.9 \pm 12.3$ & $66.6 \pm 16.3$ & \\
\hline & Median & 72.6 & 43 & 42.2 & \\
\hline & $95 \% \mathrm{CI}$ & $79.2-340.2$ & $29.2-80.6$ & $31.6-101.6$ & $P=0.007$ \\
\hline \multirow{2}{*}{10} & Median & 125.2 & 54 & 46 & \\
\hline & $95 \% \mathrm{CI}$ & $82.6-404.1$ & $27.3-77.7$ & $25.0-132.3$ & $P=0.02$ \\
\hline \multirow[t]{3}{*}{12} & Mean & $185.4 \pm 64.9$ & $131.4 \pm 44.2$ & $82.4 \pm 12.7$ & \\
\hline & Median & 90.2 & 39 & 75.3 & \\
\hline & $95 \%$ CI & $51.7-319.2$ & $39.1-223.6$ & $51.2-113.6$ & $P=0.17$ \\
\hline \multirow[t]{3}{*}{14} & Mean & $238.6 \pm 98.7$ & $121.6 \pm 34.5$ & $49.3 \pm 14.2$ & \\
\hline & Median & 74.9 & 60.0 & 43.1 & \\
\hline & $95 \% \mathrm{CI}$ & $33.7-443.4$ & $48.0-195.2$ & $14.5-84.2$ & $P=0.92$ \\
\hline
\end{tabular}

patients with AUNF alone who did not develop an IFI. If serial sampling commenced on the first day of AUNF, $78 \%$ of patients had a positive test a mean of only 1.25 days prior to the IFI clinical/radiological diagnosis, though there was a wide range of between 14 days before and 14 days after the diagnosis. Therefore some patients would be diagnosed significantly early in the evolution of their IFI. If sampling had commenced on the first day of neutropenia instead, the timing of an IFI diagnosis based on glucan concentrations could have been advanced from the first day of AUNF by approximately 5 days in $50 \%$ of those patients subsequently developing an IFI, thereby offering the opportunity for earlier therapeutic intervention. However, it is not possible to determine with certainty if the outcome for patients with IFI would have been altered by earlier treatment.

In this study all patients with AUNF received antifungal therapy, usually with liposomal amphotericin B at $3 \mathrm{mg} \mathrm{kg}^{-1}$ per day, increasing to between 5 and $10 \mathrm{mg} \mathrm{kg}^{-1}$ per day if they developed an IFI. Despite this antifungal treatment the mean cohort BG concentrations

Table 4. Performance of the BG assay

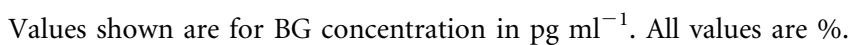

\begin{tabular}{|lcccccc|}
\hline & $\mathbf{1}$ value $\geqslant \mathbf{6 0}$ & $\mathbf{2}$ values $\geqslant \mathbf{6 0}$ & $\mathbf{1}$ value $\geqslant \mathbf{8 0}$ & $\mathbf{2}$ values $\geqslant \mathbf{8 0}$ & $\mathbf{1}$ value $\geqslant \mathbf{1 0 0}$ & $\mathbf{2}$ values $\geqslant \mathbf{1 0 0}$ \\
\hline Sensitivity & 97.4 & 92.1 & 94.7 & $\mathbf{8 6 . 8}$ & 89.5 & 81.6 \\
Specificity & 28.6 & 61.9 & 45.2 & $\mathbf{7 6 . 2}$ & 57.1 & 83.3 \\
False positives & 71.4 & 38.1 & 54.8 & $\mathbf{2 3 . 8}$ & 42.9 & 10.5 \\
False negatives & 2.6 & 7.9 & 5.3 & $\mathbf{1 3 . 2}$ & 89.5 \\
PPV & 55.2 & 68.6 & 61 & $\mathbf{7 6 . 7}$ & 85.7 \\
NPV & 92.3 & 89.7 & 90.5 & $\mathbf{8 6 . 5}$ & 81.5 \\
Accuracy & 61.3 & 76.3 & 68.8 & $\mathbf{8 1 . 3}$ & & 72.5 \\
\end{tabular}



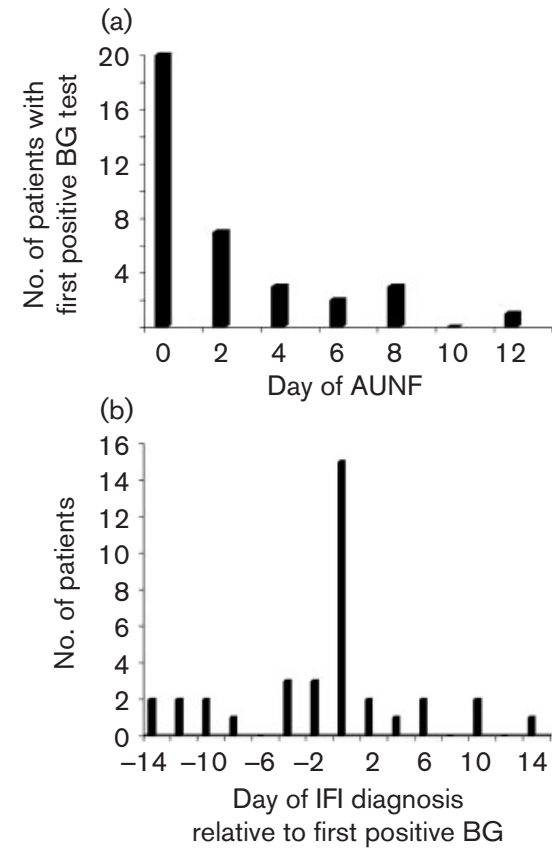

Fig. 2. (a) Day of AUNF when patients with an IFI first developed a positive BG test. (b) Day of IFI diagnosis relative to first positive $B G$ test.

were unaffected over the course of the first 10 days, a useful feature if BG sampling is performed when the patient is receiving antifungal drugs. However, the subsequent course of the BG concentrations was found to be highly variable between patients (see Figs 3 and 4). Some patients responded both clinically and with decrements in the BG concentration whilst others had persistently high levels despite antifungal treatment. Others were noted to have widely fluctuating levels. Survivorship also did not consistently correlate with the pattern of BG concentrations. The reason for the inter-patient variability is unclear and should be explored.

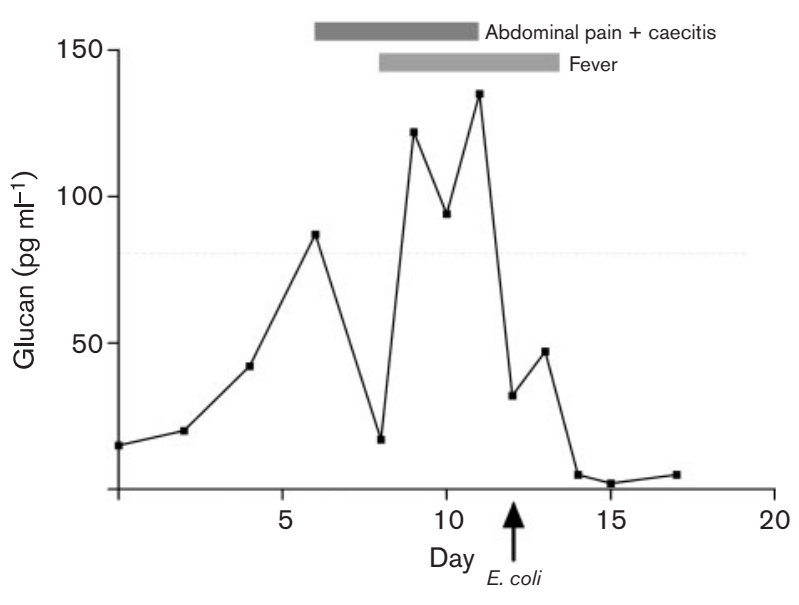

Fig. 3. Example of a patient with caecitis and abdominal pain, showing serial BG levels. The $x$-axis shows days of neutrophil count $0.1-0.5 \times 10^{9} \mathrm{I}^{-1}$. The date of $E$. coli bacteraemia is indicated by the arrow.

We found that defining a positive test for IFI as $\geqslant 2$ consecutive concentrations $\geqslant 80 \mathrm{pg} \mathrm{ml}^{-1}$ gave a sensitivity and specificity of $86.8 \%$ and $76.2 \%$, respectively, and provided a high degree of accuracy $(81.3 \%)$. Other studies used cut-off concentrations of $60 \mathrm{pg} \mathrm{ml}^{-1}$ in patients who had also received antifungal drug prophylaxis (Odabasi et al., 2004; Ostrosky-Zeichner et al., 2005). Using a lower cut-off of $60 \mathrm{pg} \mathrm{ml}^{-1}$ in our patients enhanced sensitivity but reduced specificity to an unacceptable level and gave a high false positive rate. Conversely, increasing the threshold to $100 \mathrm{pg} \mathrm{ml}^{-1}$ lowered the sensitivity slightly but increased the false negative rate to almost $20 \%$. The negative predictive value was over $83 \%$ irrespective of the cut-off value or number of tests, confirming that the assay may be useful in excluding most patients with an IFI (but see comments below on the overall usefulness of the BG test). Another recent study utilizing a single BG concentration of $\geqslant 120 \mathrm{pg} \mathrm{ml}^{-1}$ returned a sensitivity and

Table 5. Details of patients without IFI and positive BG tests

\begin{tabular}{|c|c|c|}
\hline & $\geqslant 2$ readings above $80 \mathrm{pg} \mathrm{ml}^{-1}$ & $\leqslant 1$ reading above $80 \mathrm{pg} \mathrm{ml}^{-1}$ \\
\hline No. of patients & 11 & 31 \\
\hline BG mean $\left(\mathrm{pg} \mathrm{ml}^{-1}\right)$ & $191.8 \pm 55.8$ & $44.9 \pm 3.4^{*}$ \\
\hline BG median (pg ml $\left.{ }^{-1-}\right)$ & 54 & $31^{\star}$ \\
\hline Severe mucositis or severe enterocolitis & $5 \dagger$ & $1 \ddagger$ \\
\hline No. of patients with + ve blood culture & $3 \S$ & $5 \|$ \\
\hline Proportion of patients with abnormalities & $8 / 11(72.7 \%)$ & $6 / 31(19.4 \%)$ \\
\hline
\end{tabular}

${ }^{\star} P<0.0001$.

$\dagger$ Ileitis (1 patient), colitis with perianal inflammation (2 patients), oral mucositis (2 patients).

$\ddagger$ Caecitis.

\$Coagulase-negative staphylococci (3 patients).

IICoagulase-negative staphylococci (3 patients), Escherichia coli (1 patient), Stenotrophomonas maltophilia (1 patient).

S $P=0.002$. 

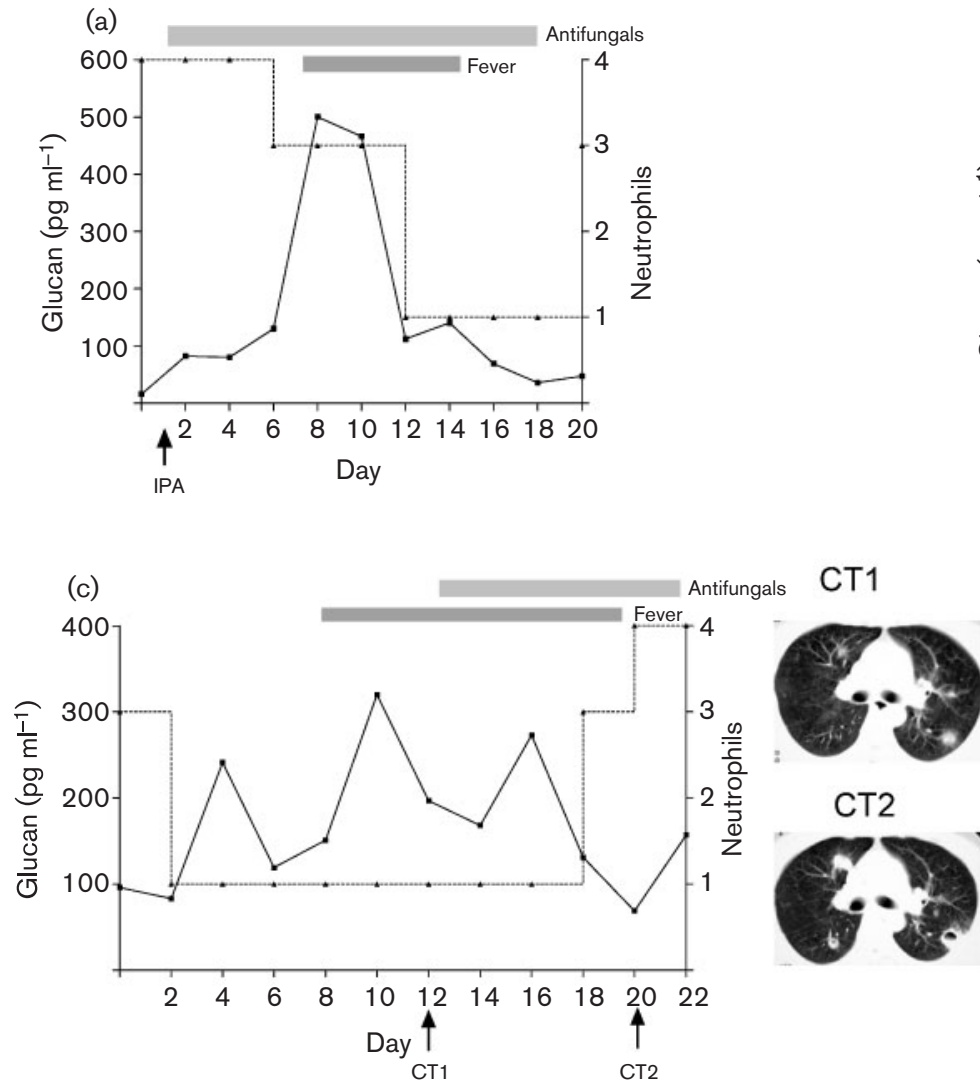

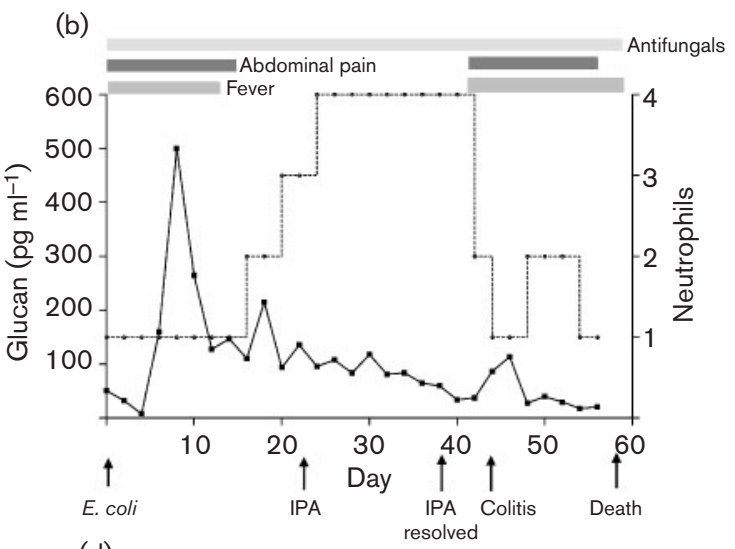

(d)

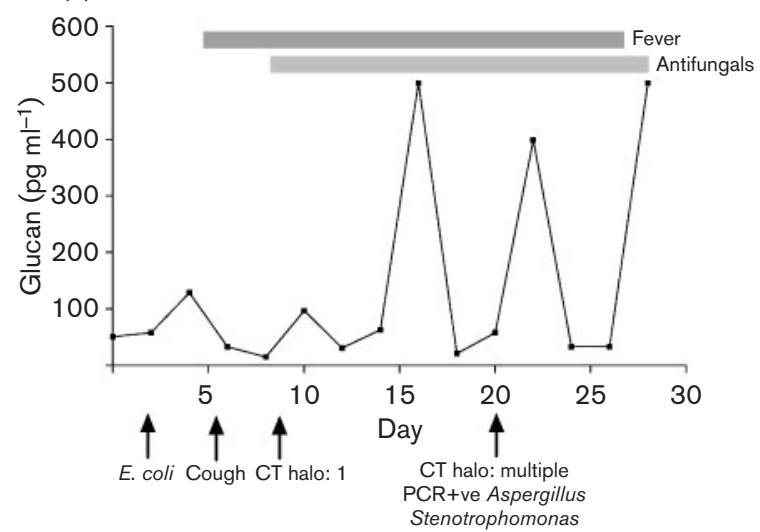

Fig. 4. Interpatient variability of serial $B G$ concentrations. See text for details. The neutrophil units indicate the range of neutrophil counts as follows $\left(\times 10^{9} \mathrm{I}^{-1}\right)$ : $1,<100 ; 2,100-500 ; 3,500-1000 ; 4,>1000$; depicted by broken lines for each day). Solid lines represent daily BG concentrations. E. coli is from blood.

specificity of $83.3 \%$ and $89.6 \%$, respectively, in neutropenic patients with invasive candidiasis (Pazos et al., 2006). These figures were increased to $100 \%$ when anti-Candida germ-tube antibodies were also included. The manufacturer's instructions for the Fungitell assay define a positive test as one value of $\geqslant 80 \mathrm{pg} \mathrm{ml}^{-1}$ but qualify this with a stated need for frequent sampling to improve diagnostic utility (Associates of Cape Cod, 2004). Indeed Odabasi et al. (2004) showed that specificity rose from 90 to $96 \%$ when two sequential positives were required. Thus the issue of cut-off and number of samples remains unclear.

It is of interest that several patients who did not develop an IFI had persistently elevated BG concentrations. Possible explanations for this phenomenon include extremely early IFI that was successfully treated by the 'empirical' antifungal therapy, or candidal translocation as a result of chemotherapy-induced enterocyte apoptosis (this is consistent with the high proportion of patients with mucositis/enterocolitis in this subgroup) that was eradicated by the antifungal drugs (Ellis, 2004; Krause et al., 1969). Finally it is known that the assay can be affected by certain biologics such as immunoglobulins, platelets and gauze (Associates of Cape Cod, 2004; Ostrosky-Zeichner et al., 2005). However, random testing of antibiotics, blood products and the sterile blood sampling tubes used in our study gave BG concentrations of under $40 \mathrm{pg} \mathrm{ml}^{-1}$ in all cases, and no patient had gauze impregnation. Therefore, although widespread contamination was unlikely, sporadic high values were still occasionally seen, making it important to base a positive result on at least two consecutive values above a predetermined cut-off.

\section{Potential applicability of real-time BG results in guiding antifungal treatment}

Clearly the usefulness of the BG assay in a cohort of patients has been shown in this study, but the interpretation of a real-time test result in any particular patient has to be individualized and viewed in the context of the test being used adjunctively with other clinical, radiological and serological data. Fig. 5 illustrates this. The initial BG concentration of $158 \mathrm{pg} \mathrm{ml}^{-1}$ together with a positive blood culture for Candida tropicalis and persistently positive PCR results for Candida spp. might justifiably guide the clinician's selection of fluconazole or caspofungin rather than the broader-spectrum liposomal amphotericin 


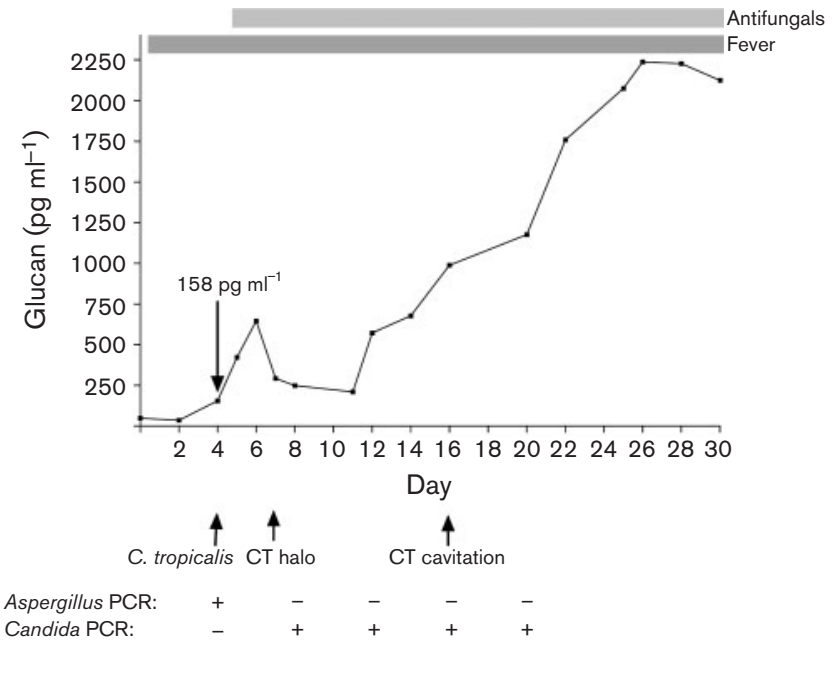

Fig. 5. This case of a double IFI illustrates the limitations in applying the results of serological testing to real-time decisions on antifungal drug therapy. See text for details. Note the very high persisting BG levels despite clinical improvement, antifungal treatment and survival (compare Fig. 3b).

B. However, subsequent CT imaging and dramatic further rises in BG concentrations confirmed the patient to have a double IFI. In retrospect the better choice of antifungal therapy would have been a polyene preparation rather than fluconazole, and this could have influenced the outcome.

Although this study was not designed to address the clinical impact of a test result as a consequence of modification of antifungal drug administration, the appropriateness of antifungal drug modification in a real-time setting can be hypothesized. If the BG results had been available to the treating physician at day 0 and day 2 of AUNF and if there had been in force a policy of discontinuation of empirical antifungal drug therapy based on negative results and vice versa, the following would be predicted. Thirty-three of 80 patients had a positive BG. Since 24/33 (73\%) patients developed an IFI, continuation of antifungal therapy would have been appropriate in those and inappropriate in the remaining $27 \%$. Of 47 with a negative BG test, $14(30 \%)$ subsequently developed an IFI; therefore discontinuation of treatment would have been inappropriate in those, but appropriate for the remaining $33(70 \%)$ who did not develop an IFI. Thus the overall usefulness of the BG test for guiding antifungal therapy based on initial BG results would be approximately $70 \%$. The effect of appropriate discontinuation of therapy would be cost-effective, but patients whose antifungal treatment would be inappropriately terminated might be at risk of an adverse outcome. Similarly, many patients who had an IFI but initial negative BG results subsequently developed positive results, but the impact of the delay in making a diagnosis on the patient outcome is unknown.

\section{Conclusion}

Alternate-day determination of BG concentrations from the first day of AUNF appears to be a useful adjunct in differentiating patients who have or will develop an IFI during the AUNF from those who have AUNF without overt IFI. By commencing sampling earlier, from the first neutropenic day, the timing of the first positive test can be advanced by 5 days. Patients with high levels of BG without an overt IFI may nevertheless have low-burden fungaemia. The impact of availability of the BG results from day 0 and day 2 of AUNF on a real-time basis might result in inappropriate alteration of antifungal treatment in $30 \%$ of patients. Our results indicate that this figure would be reduced by including results beyond days 0 and 2 . In common with many serological test results, BG concentrations should not be interpreted alone, but always in the context of a thorough clinical, microbiological and radiological assessment.

\section{REFERENCES}

Ascioglu, S., Rex, J., de Pauw, B., Bennett, J., Bille, J., Crokaert, F., Denning, D., Donnelly, J., Edwards, J. \& other authors (2002). Defining opportunistic infections in immunocompromised patients with cancer and hematopoietic stem cell transplants: an international consensus. Clin Infect Dis 34, 7-14.

Associates of Cape Cod (2004). Glucan assay for (1,3)- $\beta$-D-glucan in serum (FUNGITELL $\left.{ }^{T M}\right)$. Instructions for use.

Caillot, D., Casasnovas, O., Bernard, A., Couaillier, J. F., Durand, C., Cuisenier, B., Solary, E., Piard, F., Petrella, T. \& other authors (1997). Improved management of invasive pulmonary aspergillosis in neutropenic patients using early thoracic computed tomography scan and surgery. J Clin Oncol 15, 139-147.

Caillot, D., Couaillier, J., Bernard, A., Casasnovas, O., Denning, D., Mannone, L., Lopez, J., Couillault, G., Piard, F. \& other authors (2001). Increasing volume and changing characteristics of invasive pulmonary aspergillosis on sequential thoracic computed tomography scans in patients with neutropenia. J Clin Oncol 19, 253-259.

Coiffier, B., Lepage, E., Briere, J., Herbrecht, R., Tilly, H., Bouabdallah, R., Morel, P., Van Den Neste, E., Salles, G. \& other authors (2002). CHOP chemotherapy plus rituximab compared with CHOP alone in elderly patients with diffuse large-B-cell lymphoma. $N$ Engl J Med 346, 235-242.

De Pauw, B. E. (2005). Between over- and undertreatment of invasive fungal disease. Clin Infect Dis 41, 1251-1253.

Ellis, M. (2004). Preventing microbial translocation in haematological malignancy. Br J Haematol 125, 282-293.

EORTC International Antimicrobial Therapy Cooperative Group (1989). Empiric antifungal therapy in febrile neutropenic patients. Am J Med 86, 668-672.

Farag, S. S., Ruppert, A. S., Mrózek, K., Mayer, R. J., Stone, R. M., Carroll, A. J., Powell, B. L., Moore, J. O., Pettenati, M. J. \& other authors (2005). Outcome of induction and postremission therapy in younger adults with acute myeloid leukemia with normal karyotype: a cancer and leukemia group B study. J Clin Oncol 23, 482-493.

Fridkin, S. K. (2005). The changing face of fungal infections in health care settings. Clin Infect Dis 41, 1455-1460.

Garey, K. W., Rege, M., Pai, M. P., Mingo, D. E., Suda, K. J., Turpin, R. S. \& Bearden, D. T. (2006). Time to initiation of fluconazole 
therapy impacts mortality in patients with candidemia: a multiinstitutional study. Clin Infect Dis 43, 25-31.

Hughes, W. T., Armstrong, D., Bodey, G. P., Bow, E. J., Brown, A. E., Calandra, T., Feld, R., Pizzo, P. A., Rolston, K. V. I. \& other authors (2002). 2002 guidelines for the use of antimicrobial agents in neutropenic patients with cancer. Clin Infect Dis 34, 730-751.

Kami, M., Kishi, Y., Hamaki, T., Kawabata, M., Kashima, T., Masumoto, T., Oki, Y., Tanaka, Y., Sawada, S. \& other authors (2002). The value of the chest computed tomography halo sign in the diagnosis of invasive pulmonary aspergillosis. An autopsy-based retrospective study of 48 patients. Mycoses 45, 287-294.

Kelaher, A. (2006). Two non-invasive diagnostic tools for invasive aspergillosis: (1,3)- $\beta$-D-glucan and the galactomannan assay. Clin Lab Sci 19, 222-224.

Krause, W., Matheis, H. \& Wulf, K. (1969). Fungaemia and funguria after oral administration of Candida albicans. Lancet 1, 598-599.

Maertens, J., Verhaegen, J., Lagrou, K., van Eldere, J. \& Boogaerts, M. (2001). Screening for circulating galactomannan as a non-invasive diagnostic tool for invasive aspergillosis in prolonged neutropenic patients and stem cell transplantation recipients: a prospective validation. Blood 97, 1604-1610.

Maertens, J., Theunissen, K., Verhoef, G., Verschakelen, J., Lagrou, K., Verbeken, E., Wilmer, A., Verhaegen, J., Boogaerts, M. \& van Eldere, J. (2005). Galactomannan and computed tomography-based preemptive antifungal therapy in neutropenic patients at high risk for invasive fungal infection: a prospective feasibility study. Clin Infect Dis $\mathbf{4 1}$, $1242-1250$.

Martino, R. \& Viscoli, C. (2005). Empirical antifungal therapy in patients with neutropenia and persistent or recurrent fever of unknown origin. Br J Haematol 132, 138-154.

Medical Research Council Working Party on Leukemia in Adults (2006). Medical Research Council Acute Lymphoblastic Leukemia Trial XII (ULALL XII), Version 4.1, February 2006. Joint Trial with Eastern Cooperative Oncology Group (ECOG) - E2993. Protocol for
Adults with ALL. Modified for Intrathecal Therapy and Philadelphia Positive ALL, July 2002. Revised May 2005, February 2006. Available at http://www.ctsu.ox.ac.uk/projects/ukallxii/

Odabasi, Z., Mattiuzzi, G., Estey, E., Kantarjian, H., Saeki, F., Ridge, R. J., Ketchum, P. A., Finkelman, M. A., Rex, J. H. \& OstroskyZeichner, L. (2004). $\beta$-D-Glucan as a diagnostic adjunct for invasive fungal infections: validation, cut-off development, and performance in patients with acute myelogenous leukemia and myelodysplastic syndrome. Clin Infect Dis 39, 199-205.

Ostrosky-Zeichner, L., Alexander, B. D., Kett, D. H., Vasquez, J., Pappas, P. G., Saeki, F., Ketchum, P. A., Wingard, J., Schiff, R. \& other authors (2005). Multicenter clinical evaluation of the $(1,3)-\beta$ D-glucan assay as an aid to diagnosis of fungal infections in humans. Clin Infect Dis 41, 654-659.

Pazos, C., Moragues, M., Quindos, G., Ponton, J. \& del Palacio, A. (2006). Diagnostic potential of $(1 \rightarrow 3)$ - $\beta$-D-glucan and anti-Candida albicans germ tube antibodies for the diagnosis and therapeutic monitoring of invasive candidiasis in neutropenic adult patients. Rev Iberoam Micol 23, 209-215.

Pfeiffer, C. D., Fine, J. P. \& Safdar, N. (2006). Diagnosis of invasive aspergillosis using a galactomannan assay: a meta-analysis. Clin Infect Dis 42, 1417-1427.

Pizzo, P. A., Robichaud, K. J., Gill, F. A. \& Witebsky, F. G. (1982). Empiric antibiotic and antifungal therapy for cancer patients with prolonged fever and granulocytopenia. Am J Med 72, 101-107.

Verweij, P. E., Figueroa, J., van Burik, J., Holdom, M. D., Dei-Cas, E., Gomez, B. L. \& Mendes-Giannini, M. (2000). Clinical applications of non-culture based methods for the diagnosis and management of opportunitic and endemic mycoses. Med Mycol 38, 161-171.

Walsh, T. J., Finberg, R. W., Arndt, C., Hiemenz, J., Schwartz, C., Bodensteiner, D., Pappas, P., Seibel, N., Greenberg, R. N. \& other authors (1999). Liposomal amphotericin B for empirical therapy in patients with persisting fever and neutropenia. N Engl J Med 340, 764-771. 